\title{
XLVI. On the foundations of the kinetic theory of gases
}

\section{Prof. Tait}

To cite this article: Prof. Tait (1886) XLVI. On the foundations of the kinetic theory of gases , Philosophical Magazine Series 5, 21:131, 343-348, DOI: 10.1080/14786448608627855

To link to this article: http://dx.doi.org/10.1080/14786448608627855

曲 Published online: 08 Jun 2010.

Submit your article to this journal $\pi$

Џ Article views: 11

Q View related articles $\square$

Citing articles: 1 View citing articles 5 
XLVI. On the Foundations of the Kinetic Theory of Gases. By Prof. TaIT *.

THE attempt to account for the behaviour of gases by at1 tributing continuous pressure to exceedingly numerous, but nearly infinitesimal, impacts on the containing vessel is probably very old. It certainly occurs, with some little development, in Hooke's tract of 1676 , "Lectures de potentia restitutiva, or of Spring"; and, a little more fully developed, in the Hydrodynamica of D. Bernouilli, 1738. It was recalled to notice in 1847 by Herapath in his Mathematical Physics, and applied, in 1848, by Joule to the calculation of the average speed of the particles in a mass of hydrogen at $0^{\circ} \mathrm{C}$.

In and after 1857 Clausius greatly improved the theoretical treatment of the problem by taking account of the mutual impacts of the particles and the rotations which they communicate to one another, at the same time introducing (but only to a limited extent) the statistical method. In this series of papers we find the first hint of the length of the mean free path of a particle, and the explanation of the comparative slowness of the process of diffusion of one gas into another. But throughout it is assumed, so far as the calculations are concerned, that the particles of a gas are all moving with equal speeds.

In this Magazine for 1860 Clerk-Maxwell published his papers on the "Collision of Elastic Spheres," which had been read to the British Association in the previous year. In this very remarkable investigation we have the first attempts at a numerical determination of the length of the mean free path. These are founded on the observed rate of diffusion of gases into one another ; and on the viscosity of gases, which here first received a physical explanation. The statistical method is allowed free play, and consequently the law of distribution of speed among the impinging particles is investigated, whether these be all of one kind or a mixture of two or more kinds. In the ardour of his research, Maxwell here and there contented himself with very incomplete proofs (we can scarcely call them more than illustrations) of some of the most important of his results. This is specially the case with the investigation of the law of ultimate partition of energy in a mixture of smooth spherical particles of two different kinds. He obtained,

* Abstract of Prpers read to the Royal Society of Edinburgh, Dec. 7th, 1885, and subsequently: communicated (by permission of the Council) at the request of sir W. Thomson. 
in accordance with the so-called Lavo of Avogadro, the result that the average energy of translation is the same per particle in each system; and he extended this in a corollary to a mixture of any number of different systems. This proposition, if true, is of fundamental importance. It has since been extended by Boltzmann and others to cases in which the individual particles are no longer supposed to be hard smooth spheres, but complex systems having great numbers of degrees of freedom. And it is stated, as the result of a process which is rather of the nature of playing with symbols than of reasoning by consecutive steps, that in such groups of systems the ultimate state will be a partition of the whole energy in equal shares among the classes of degrees of freedom which the individual particle-systems possess. This, it accepted as true, at once raises a formidable objection to the kinetic theory. For there can be no doubt that each individual particle of a gas has a very great number of degrees of freedom besides the six which it would have if rigid:- the examination of its spectrum while incandescent proves this at once. But if all these degrees of freedom are to share the whole energy (on the average) equally among them, the results of theory will no longer be cunsistent with our experimental knowledge of the relations between the two specific heats of a gass.

Hence it is desirable that Maxwell's proof of his fundamental Theorem should be critically examined, and improved where it may be found defective. If it be shown in this process that certain preliminary conditions are absolutely necessary to the proof even of Maxwell's Theorem, and if these cannot be granted in the more general case treated by Boltzmann, it is clear that Boltzmann's Theorem must be abandoned.

1. The chief features, besides too great conciseness, in respect of which Maxwell's proof is objectionable are :-

(a) $\mathrm{He}$ assumes that the transference of energy from one system to the other can be calculated from the results of a single impact between particles, one from each system, each having the average translational energy of its system.

Thus (so far as this step is concerned) the distribution of energy in each system may be any whaterer.

(b) In this typical impact the velocities of the impinging spheres are taken as at right angles to one ancther, so that the relative speed may be that of mean square as between the particles of the two systems. The result obtained is fallacious because, in general, the directions of motion after impact are found not to be at right angles to one another, as they would certainly be (on account of the perfect reversibility of the motions) were this really a typical impact. 
(c) Maxwell proceeds as if every particle of one system impinged upon one of the other system at each stage of the process- $i$. e. he calculates the transference of energy as if each pair of particles, one from each system, had simultaneously a typical impact. This neglect of the immensely greater number of particles which either had no impact or impinged on others of their own group makes the calculated rate of equalization far too rapid.

(d) Attention is not called to the fact that impacts between particles are numerous in proportion to their relative speed, nor is this consideration introduced in the calculations.

(e) Throughout the investigation each step of the process of avernging is performed (as a rule) before the expressions are ripe for it.

2. In seeking for a proof of Maxwell's Theorem, I found it absolutely essential to the application of the statistical method to premise :mixed.

(A) That the particles of the two systems are thoroughly

(B) That, in any region containing a very large number of particles, the partieles of each kind acquire and maintain the error-law distribution of velocities. This will be referred to as the "special" state. The disturbances of this arrangement produced in either system by impacts on members of the other are regarded as being promptly repaired by means of the internal collisions in the system itself. This is the sole task assigned to these internal collisions. We assume that they accomplish it, so we need not further allude to them.

[The warrant for these assumptions is sought in the fact that only a small fiaction of the whole particles are at any instant in collision ; $i$. e. that each particle advances, on the average, through a considerable multiple of its diameter before it encounters another.]

(C) That there is perfectly free access for collision between each pair of particles, whether of the same or of different systems ; and that, in the mixture, the number of particles of one kind is not overwhelmingly greater than that of the other kind.

[This is one of the essential points which seem to be wholly ignored by Boltzmann and his commentators. There is no proof given by them that one system, while regulating by its internal collisions the distribution of energy among its own members, can also by impacts regulate the distribution of energy among the members of another system, when these are not free to collide with one another. In fact, if (to take 
an extreme case) the particles of one system were so small, in comparison with the average distance between any two contiguous ones, that they practically had no mutual collisions, they would behave towards the particles of another system much as Le Sage supposed his ultra-mundane corpuscles to behave towards particles of gross matter. Thus they would merely alter the apparent amount of the molecular forces between the particles of a gas. And it is specially to be noted that this is a question of effective diameters merely, and not of masses.]

3. With these assumptions we may proceed as follows:-Let $P$ and $Q$ be the masses of particles from the two systems respectively; and when they impinge, let $\mathrm{u}, \mathrm{v}$ be their velocitycomponents measured towards the same parts along the line of centres at impact. If these velocities become, after impact, $u^{\prime}, v^{\prime}$ respectively, we have at once

$$
P\left(u^{\prime}-u\right)=-\frac{2 P Q}{P+Q}(u-v)=-Q\left(v^{\prime}-v\right) ;
$$

an immediate consequence of which is

$$
\begin{aligned}
P\left(u^{\prime 2}-u^{2}\right)=-\frac{4 P Q}{(P+Q)^{2}}( & \left.P u^{2}-Q v^{2}-(P-Q) u v\right) \\
& =-Q\left(v^{\prime 2}-v^{2}\right) .
\end{aligned}
$$

Hence, denoting by a bar the average value of a quantity, we see that transference of energy between the systems must cease when

$$
\overrightarrow{\mathrm{Pu}^{2}}-\mathrm{Q} \overline{\mathrm{v}^{2}}-(\mathrm{P}-\mathrm{Q}) \overline{\mathrm{uv}}=0, \quad \cdot \quad \cdot \quad \cdot
$$

and the question is reduced to finding these averages.

I thought at first that $\overline{u v}$ might be assumed to vanish, and that $\overline{u^{2}}$ and $\overline{v^{2}}$ might each be taken as one third of the meansquare speed in its system. This set of suppositions would lead to Maxwell's Theorem at once. But it is clear that, when two particles have each a given velocity, they are more likely to collide when they are moving towards opposite parts than when towards the same parts. Hence $\overline{\mathrm{uv}}$ must be an essentially negative quantity, and therefore $P \overline{u^{2}}$ necessarily less than $\overline{\mathrm{Qv}^{2}}$, if $\mathrm{P}$ be greater than $\mathrm{Q}$. Thus it seemed as if the greater masses would have on the average less energy than the smaller.

4. But these first impressions are entirely dissipated when we proceed to calculate the average values. For it is found 
that if we write (1) in the form

$$
\overline{P u^{2}-u v}-\overline{Q v^{2}-u v}=0, \text {. . . . . }
$$

the terms on the left are equal multiples of the average energy of a $\mathrm{P}$ and of a $\mathrm{Q}$ respectively. Thus Maxwell's Theorem is rigorously true, though in a most unexpected manner. There mast surely be some extremely simple and direct mode of showing that $\overline{\mathbf{u}^{2}-\mathbf{u v}}$ is independent of the mean-square speed of the system of Qs. Meanwhile I give the heads of the investigation by which I arrived at the result just stated.

5 . It is shown that, if a particle move constantly with velocity $v$ among a system of other particles which are in the "special" state, the fraction of the whole of its encounters which take place with particles whose velocity is from $v_{1}$ to $v_{1}+d v_{1}$, and whose directions of motion are inclined to its direction at angles from $\beta$ to $\beta+d \beta$, is proportional to

where

$$
\epsilon^{-q v_{1}^{2}} v_{1}^{2} v_{0} d v_{1} \sin \beta d \beta \text {; }
$$

$$
v_{0}=\sqrt{v^{2}+v_{1}^{2}-2 v v_{1} \cos \beta}
$$

is the relative velocity, and $3 / 2 g$ is the mean-square speed of the spheres of the system.

The line of centres at impact depends for its positions upon the condition that the line of relative motion of the centre of one of the impinging particles may pass, with equal probability, perpendicularly through all equal areas of a diametral plane of the sphere, whose radius is the sum of the radii, and which is concentric with the other particle.

The number of particles of the first system which have speeds between $v$ and $v+d v$ is proportional to

$$
\epsilon^{-p v^{2}} v^{2} d v
$$

where the mean-square speed of the system is $3 / 2 p$.

Taking these things into account, it is found that

and

$$
\overline{\mathrm{u}^{2}}=\frac{p+2 q}{2 p(p+q)}
$$

so that

$$
\overrightarrow{\mathrm{uv}}=-\frac{1}{2(p+q)},
$$

$$
\overline{\mathrm{u}^{2}-\overline{\mathrm{uv}}}=\frac{1}{p},
$$

which gives the result stated above. 
348 On the Houndations of the Kinetic Theory of Gases.

These values are deduced from particular cases of the curious expression

$$
\begin{gathered}
\int_{0}^{\infty} e^{-p x^{2}} x d x\left(\int_{0}^{x} e^{-q y^{2}} y d y\left(\overline{x+y^{2 n+1}}-\overline{x-y^{2 n+1}}\right)\right. \\
\left.+\int_{x}^{\infty} \epsilon^{-q y 2} y d y\left(\overline{y+x^{2 n+1}}-\overline{y-x^{2 n+1}}\right)\right) \\
=\frac{\sqrt{\pi}}{4} n !(2 n+1) \frac{(p+q)^{\frac{2 n-1}{2}}}{(p q)^{n+1}},
\end{gathered}
$$

which, in its turn, may be made to depend upon the following pair of fundamental theorems :-

$$
\begin{aligned}
& \int_{0}^{\infty} \epsilon^{-p x^{2}} x d x \int_{0}^{x} \epsilon^{-q y^{2}} d y=\frac{\sqrt{ } \pi}{4 p \sqrt{p+q}}, \\
& \int_{0}^{\infty} \epsilon^{-p x^{2}} d x \int_{x}^{\infty} \epsilon^{-q y^{2}} y d y=\frac{\sqrt{ } \pi}{4 q \sqrt{p+q}} .
\end{aligned}
$$

6. Another question of importance in the theory regards the proper definition of the Mean Free Path. From the point of view adopted by Maxwell, and since taken by Meyer, Watson, and others, the mean freo path in a system of equal spheres is

average speed of a sphere

. average number of collisions per sphere per second

It seems to be more in accordance with the usual sense of the word "mean" to define the mean path as the sum of the products of the mean path for each speed into the chance of that speed. And those who adopt the above deviation from the ordinary usage must, I think, face the question,-Why not deviate in the opposite direction, and define the mean path as (average time of describing a free path) $\times$ (average speed)?

The numerical values deduced from these three definitions bear to one another, in the above order, the ratios

$$
0.707: 0.677: 0.647 \text {; }
$$

unit being the length of the mean path of a particle (whatever its speed) if all the others were reduced to rest, and evenly distributed throughout the space which they occupied while in motion. 